\title{
Review
}

\section{New knowledge of the mechanisms of sorafenib resistance in liver cancer}

\author{
Yan-jing ZHU ${ }^{1, \#}$, Bo ZHENG ${ }^{1, \#}$, Hong-yang WANG ${ }^{1,2, *}$, Lei CHEN ${ }^{1,2, *}$ \\ ${ }^{1}$ International Co-operation Laboratory on Signal Transduction, Eastern Hepatobiliary Surgery Institute, Second Military Medical \\ University, Shanghai 200438, China; ${ }^{2}$ National Center for Liver Cancer, Shanghai 201805, China
}

\begin{abstract}
Sorafenib is an oral multikinase inhibitor that suppresses tumor cell proliferation and angiogenesis and promotes tumor cell apoptosis. It was approved by the FDA for the treatment of advanced renal cell carcinoma in 2006, and as a unique target drug for advanced hepatocellular carcinoma (HCC) in 2007. Sorafenib can significantly extend the median survival time of patients but only by 3-5 months. Moreover, it is associated with serious adverse side effects, and drug resistance often develops. Therefore, it is of great importance to explore the mechanisms underlying sorafenib resistance and to develop individualized therapeutic strategies for coping with these problems. Recent studies have revealed that in addition to the primary resistance, several mechanisms are underlying the acquired resistance to sorafenib, such as crosstalk involving PI3K/Akt and JAK-STAT pathways, the activation of hypoxia-inducible pathways, and epithelial-mesenchymal transition. Here, we briefly describe the function of sorafenib, its clinical application, and the molecular mechanisms for drug resistance, especially for HCC patients.
\end{abstract}

Keywords: sorafenib; hepatocellular carcinoma; targeted therapy; drug resistance; individualized treatment

Acta Pharmacologica Sinica (2017) 38: 614-622; doi: 10.1038/aps.2017.5; published online 27 Mar 2017

\section{Introduction}

Since the molecular revolution of the 1980s, knowledge of the etiology of cancer has increased dramatically, leading to the discovery and development of targeted therapies tailored to inhibit cancer-specific pathways. Among the numerous molecular targeted drugs, sorafenib, an oral multi-target kinase inhibitor that is also known as Nexavar, was developed by the Bayer and Onyx companies. Due to its effect on renal cell carcinoma and hepatocellular carcinoma, sorafenib has been clinically approved for the treatment of advanced renal cell carcinoma and hepatocellular carcinoma $(\mathrm{HCC})^{[1]}$. Moreover, several clinical trials, including those for NSCLC ${ }^{[2]}$, metastatic thyroid cancer ${ }^{[3]}$, steroid-refractory prostate cancer ${ }^{[4]}$, and metastatic breast cancer ${ }^{[5]}$, with sorafenib as the potential therapeutic strategy are now ongoing.

Being a multi-target kinase inhibitor, sorafenib can block tumor cell proliferation by inhibiting the activity of Raf-1, B-Raf and kinases in the Ras/Raf/MEK/ERK signaling path-

\footnotetext{
\# These authors contributed equally to this work.

${ }^{*}$ To whom correspondence should be addressed.

E-mail chenlei@smmu.edu.cn (Lei CHEN); hywangk@vip.sina.com (Hong-yang WANG)

Received 2016-11-21 Accepted 2017-01-19
}

way. Additionally, sorafenib can inhibit angiogenesis through targeting of the hepatocyte factor receptor (c-Kit), Fms-like tyrosine kinase (FLT-3), vascular endothelial growth factor receptor (VEGFR)-2, VEGFR-3, platelet-derived growth factor receptor (PDGFR- $\beta$ ) and other tyrosine kinases ${ }^{[6,7]}$ (Figure 1). Preclinical studies have also found that sorafenib is effective in various tumor cells, such as breast cancer MDA-MB-231 (containing G463V b-raf and k-ras gene mutations), melanoma LOX, and pancreatic BxPC3 cells, as well as colon cancer HCT116, DLD-1 and Colo-205 cells ${ }^{[8,9]}$ and other tumor cell lines.

Although sorafenib has opened a window of hope after decades of searching for effective agents to treat HCC, the overall outcomes are far from satisfactory. Its side effects have hampered its use; adverse events that have been reported ${ }^{[10]}$ in patients receiving sorafenib are predominantly gastrointestinal, constitutional, or dermatologic in nature, including diarrhea, weight loss, and hand-foot skin reactions. In severe cases, it can lead to hypertension, abdominal pain and even discontinuation of therapy. In addition, due to the genetic heterogeneity of HCC, some patients are initially resistant to sorafenib, which has led to the identification of predictive biomarkers for primary resistance to sorafenib. It has recently been reported that basal pERK levels, JNK and VEGFA may 


\section{Multi-kinase inhibition by Sorafenib}

\section{Multi-kinase inhibition $(X)$ upstream and downstream in tumor proliferation and angiogenic pathways serves to block tumor growth.}
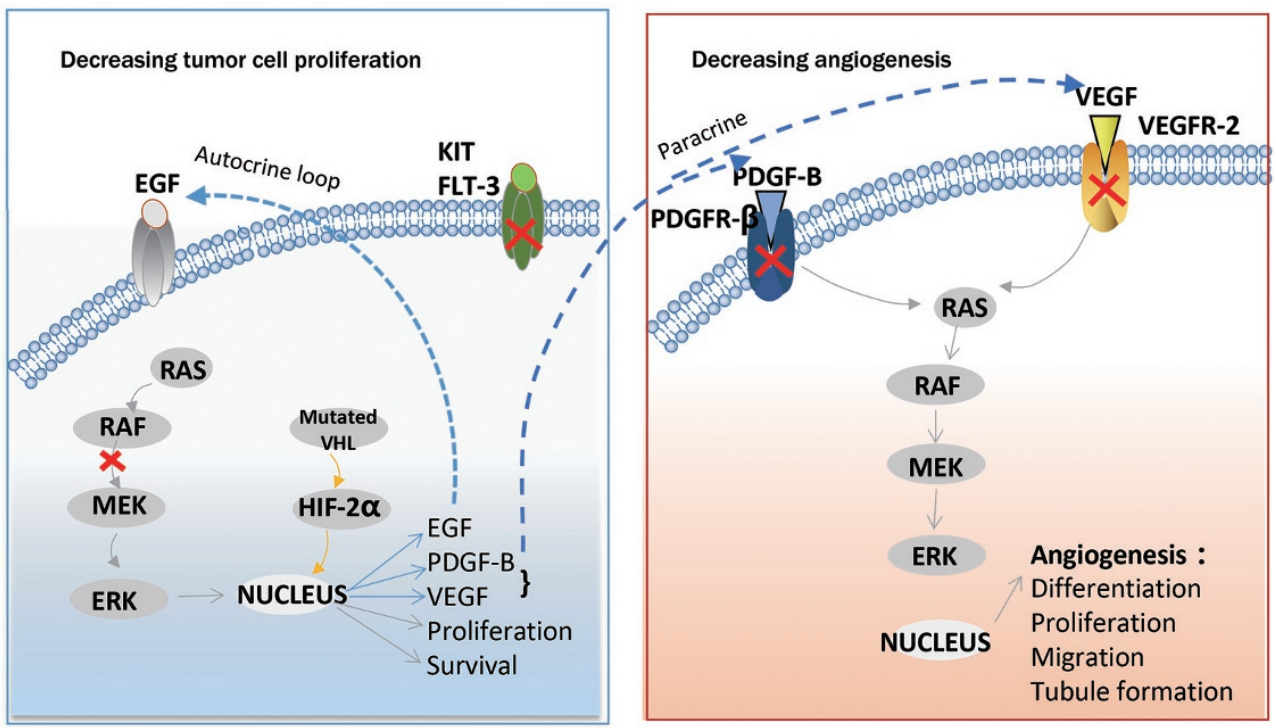

Figure 1. Cellular targets of sorafenib. Sorafenib blocks receptor tyrosine kinase signaling (VEGFR, PDGFR, c-Kit and RET) and inhibits downstream Raf serine/threonine kinase activity to prevent tumor growth by anti-angiogenic, antiproliferative and/or pro-apoptotic effects ${ }^{[10]}$ (from Bayer website).

be candidate predictors of sorafenib response in HCC. The acquired resistance to sorafenib has also drawn attention. Several mechanisms are involved in the acquired resistance to sorafenib, such as crosstalk involving the PI3K/Akt and JAK-STAT pathways, the activation of hypoxia-inducible pathways and epithelial-mesenchymal transition, as well as others. Here, we attempt to describe the function of sorafenib, its clinical application, and molecular mechanisms for drug resistance, especially for HCC patients.

\section{Clinical application in HCC Monotherapy}

Hepatocellular carcinoma (HCC) is the most common primary malignant tumor of the liver; its annual diagnosis rate ranks fifth in cancers around the globe, and it is also the third major leading cause of cancer-related death ${ }^{[11]}$. With nearly 700000 new cases every year, China accounts for over $50 \%$ of newly diagnosed HCC worldwide ${ }^{[12]}$. Because the number of HBV carriers (>120000000) in China is large, chronic hepatitisinduced cirrhosis and hepatocyte malignant transformation is the major pathogenic factor for HCC. Due to the lack of effective targeted drugs, there are no standard adjunct therapies following surgical resection at present. According to previous clinical trials, sorafenib displays significant efficacy in the treatment of advanced HCC patients.

In 2008, the SHARP (Sorafenib HCC Assessment Randomized Protocol) trial, a randomized controlled phase III trial for the treatment of HCC in international multi-centers was published in the New England Journal of Medicine ${ }^{[1]}$. In total, 602 advanced HCC patients who had not received systematic treatment were randomly divided into two groups: one with sorafenib treatment of doses of $400 \mathrm{mg}$, bid and the other as placebo. The results showed that sorafenib was significantly associated with a higher median OS (10.7 versus 7.9 months; $P=0.0006)$ and a higher median TTP (5.5 versus 2.8 months; $P=0.000007)$ compared with the placebo group. This showed that sorafenib as a first-line drug for advanced HCC could prolong the median OS and radiological progression by 3 months compared with the placebo ${ }^{[13]}$ (Figure 2). Interestingly, Cheng et $a l^{[13]}$ reported that the Asian-Pacific portion of the phase III

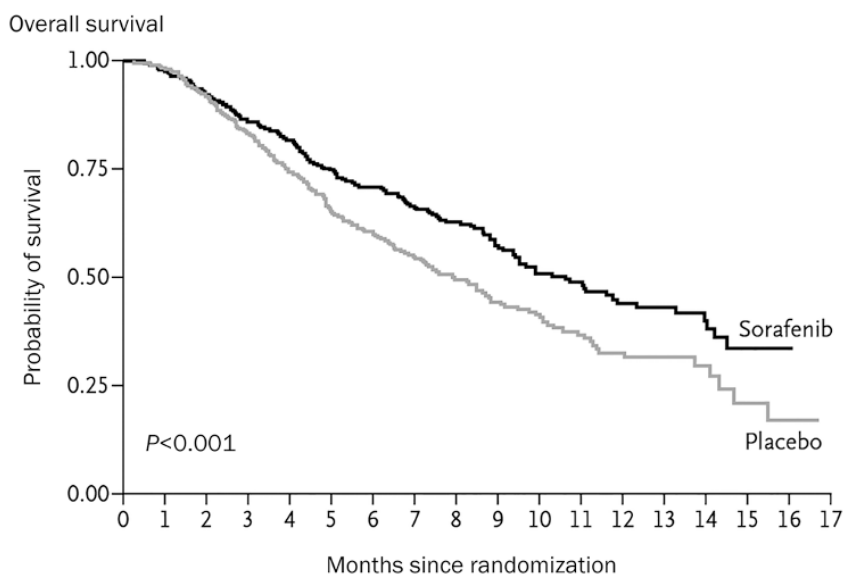

No at risk

Sorafenib $299290270249234213200172 \quad 140 \quad 111 \quad 89 \quad 68 \quad 48 \quad 37 \quad 24 \quad 7 \quad 1 \quad 0$

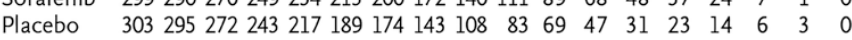

Figure 2. Kaplan-meier analysis of overall survival in the SHARP trial ${ }^{[1]}$. 
double-blind, randomized, controlled clinical trial of sorafenib treatment also demonstrated that sorafenib could prolong the OS of advanced HCC patients by 2.3 months, consistent with the SHARP trial (Figure 3). Based on this result, the FDA approved sorafenib for the treatment of unresectable HCC.

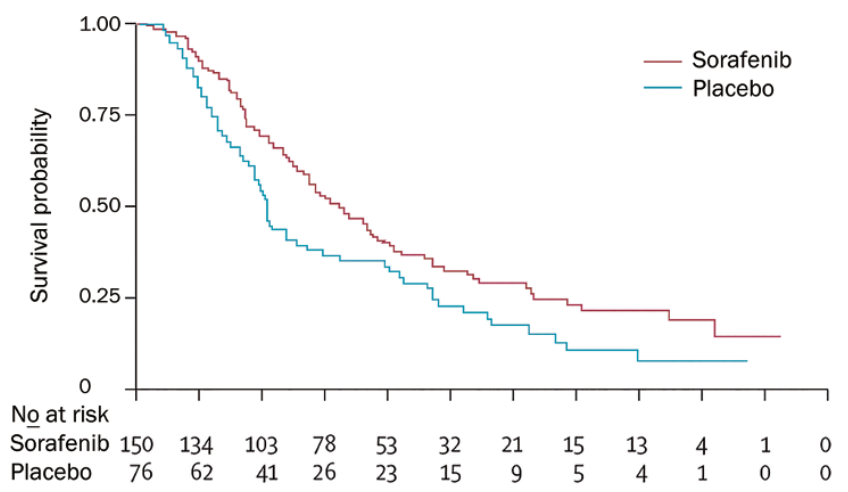

Figure 3. Kaplan-meier analysis of overall survival in the Asian-Pacific trial $^{[13]}$.

\section{Combination with other drugs}

Although single-agent sorafenib has an effect on HCC, its adverse effects, such as rash, diarrhea, high blood pressure, and hand-foot syndrome, limit high-dosage use of sorafenib ${ }^{[14]}$. Therefore, it is necessary to combine sorafenib with other drugs to lower its onset concentration. Additionally, although sorafenib is a multi-targeted agent, it has a relatively single mechanism compared with the complex growth mechanism of tumors and needs to be used with other anti-tumor agents. Both basic and clinical studies suggest that sorafenib in combination with other medicines has a favorable effect and provides a new approach for clinical tumor treatment.

Capecitabine is an oral cytotoxic agent that has selective activity against tumor cells and can be transformed to a cytotoxic drug called 5-fluorouracil by adenylate in tumor tissues to inhibit the phosphorylation of Akt and induce the expression of RKIP. Due to the over-expression of multi-resistant and P-glycoprotein genes in HCC cells, they may have potent resistance to capecitabine ${ }^{[15]}$. Sui et $a l^{[16]}$ randomly divided 42 HCC patients into 2 groups, one subjected to a combination of sorafenib and capecitabine at doses of S200 $\mathrm{mg}$ bid+C1500 $\mathrm{mg} /\left(\mathrm{m}^{2} \cdot \mathrm{d}\right)$ and the other subjected to monotherapy with capecitabine at the same dose. Those results showed that the combination therapy could prolong the TTP (6.8 versus 4.3 months) and MST (10.9 versus 7.2 months) without additional toxic reactions. In addition, Awada et al ${ }^{[17]}$ designed a series of combined therapies with sorafenib and capecitabine and found that the combination of sorafenib and capecitabine at doses of $400 \mathrm{mg}$ bid and $850 \mathrm{mg} / \mathrm{d}$ bid, respectively, achieved a higher tumor inhibition rate with fewer adverse effects in the treatment of advanced solid tumors, including HCC.

Doxorubicin, a type of anthracycline, can intercalate into
DNA and inhibit the synthesis of nucleic acids, and it has broad-spectrum activity against tumors. Abou-Alfa et al phase II clinical trial ${ }^{[18]}$ suggested that the combination of sorafenib with doxorubicin could synergistically inhibit the proliferation of tumor cells and the formation of new blood vessels in tumor nodules through the "hyperchromic effect." Furthermore, it is necessary to properly optimize the dose intensity of doxorubicin because the AUC (the area under the curve) and $C_{\max }$ (maximum concentration) of doxorubicin in the human body are limited ${ }^{[19]}$.

Fluorouracil has the ability to inhibit metabolites. It is transformed into 5F-dUMP in tumor cells and can inhibit thymidylate synthase (TS), disturb the synthesis of DNA, and attenuate the proliferation of tumor cells. Petrini et al initiated a study to evaluate the clinical potential of a combination drug of sorafenib and low-dose fluorouracil in the treatment of 38 HCC patients, which showed that the MPF and OS were 9.6 and 12.2 months, respectively ${ }^{[20]}$. The drug had a positive response and could effectively lower the dose intensity of fluorouracil and therefore reduce adverse effects.

Additionally, studies of the combination of sorafenib and uracil-tegafur ${ }^{[21]}$ or octreotide ${ }^{[22]}$ reported a better curative effect when they are used as a combination therapy than when they are used as a monotherapy, which could reduce the dosage of sorafenib and the rate of side effects.

\section{Combination with traditional treatment}

According to recent studies, combining sorafenib with traditional treatment also has potential clinical utility. Sorafenib can inhibit the synthesis of VEGF and the formation of new blood vessels in tumors after surgical resection of HCC. It also has been used to prevent the recurrence of tumors after liver transplantation for HCC beyond the Milan criteria and prolong the OS of patients with recurrent tumors. RFA (radiofrequency ablation) is the most common clinical treatment for localized physical ablation therapy of HCC, and it has several advantages such as minimal invasion, quick recovery rate, wide application and better repeatability ${ }^{[23]}$. Sun and his colleagues ${ }^{[24]}$ found that the combination of RFA and sorafenib could extend the median progression-free survival time further than mono-RFA treatment for advanced-stage HCC (7.8 versus 4.6 months). Furthermore, patients receiving the drug combination experienced an improvement in survival rate within 6 months $(80.0 \%$ versus $61.2 \%)$ and 12 months $(53.3 \%$ versus $30.4 \%$ ) compared with the monotherapy. TACE (transarterial chemoembolization) is a well-used technique to treat advanced HCC patients with no opportunity for surgical resection. Chao et al ${ }^{[25]}$ conducted a phase II clinical trial with combination treatment of sorafenib and TACE for unresectable HCC and found that the disease control rate was up to $91.2 \%$ and that the combination treatment considerably increased the survival time for moderate- and late-stage HCC patients.

However, one international study published recently in Lancet Oncology concerning a randomized phase III clinical trial $(\mathrm{STORM})^{[26]}$ surprised scholars both domestically and abroad. This trial involved 1114 HCC patients who had already under- 
gone resection and ablation. The STORM trial randomized them into groups taking sorafenib or the placebo at $400 \mathrm{mg}$ as adjuvant therapy twice a day for 4 years. The result showed that the RFS rates of the two groups were 33.4 and 33.8 months, which were considered statistically non-significant. Their OS also demonstrated no difference. Thus, sorafenib cannot improve the RFS and OS of HCC patients who have already undergone resection and ablation. Additionally, as patients have different hepatic injuries, adjuvant therapy with sorafenib would cause frequent toxic reactions and intolerance.

The results of the STORM trial challenged a wide range of oncologists and raised many questions regarding the specific efficiency and target patients of sorafenib. The SHARP and Asian-Pacific trials were all targeted at advanced HCC patients who had not received systematic treatment to evaluate the efficiency of sorafenib. However, the STORM trial targeted patients who intended to receive curative resection or localized ablation and those who were at an intermediate or high risk of recurrence to evaluate the efficiency of sorafenib as an adjuvant therapy to prevent the recurrence of HCC. The results showed that sorafenib as an adjuvant treatment did not improve the RFS and OS of HCC patients who had previously received curative treatment. Those three trials suggested that more clinical trials with different subpopulations of HCC patients and potential molecular classification markers are needed to further explore the practical effect of sorafenib on HCC. Additionally, there are ongoing studies investigating the combination of sorafenib and TACE; the efficacy of this treatment has not yet been demonstrated ${ }^{[27,28]}$ (Table 1). Alternatively, clinical trials of adjuvant treatment with sorafenib in combination with ECOG1208 (NCT01004978), TACE-2 (NCT01324076) and TACTICS (NCT01217034) are still ongoing (Table 1). Further research and joint efforts from clinicians are also needed to implement combination therapy with sorafenib and traditional treatment for advanced HCCs.

\section{Mechanisms of drug resistance}

Although sorafenib seems to be effective in prolonging median survival time with limited side effects in HCC patients, it may cause resistance in many patients, which has become an obstacle to extending the overall survival time for HCC patients. In addition, the results of the STORM trial suggest that sorafenib cannot prolong the survival of liver cancer patients who have accepted radical treatment, which posed a challenge to the application of sorafenib. At present, there are studies on the mechanisms of drug resistance to sorafenib, which may include the following aspects.

\section{Primary resistance}

Because of the genetic heterogeneity of HCC, some HCC cells and patients are initially resistant to sorafenib, which is termed primary resistance ${ }^{[29]}$. However, the exact mechanism remains unclear.

EGFR is the expression product of the proto-oncogene c-erbB1, which resides on the surface of epithelial cells. After binding with ligand, EGFRs can activate a series of downstream signaling pathways, thus regulating cell growth and proliferation. More than half of HCC patients have EGFR over-expression and abnormal activation ${ }^{[30]}$. Studies have shown that the abnormal activation of EGFR/HER3 and the overexpression of both EGFR and its ligand (especially double adjustable ligand) can inhibit the antitumor effect of sorafenib. Nonetheless, when the phosphorylation of EGFR/HER-3 was inhibited by RNA interference and combined with sorafenib, the anti-tumor proliferation and pro-apoptotic abilities of sorafenib were improved ${ }^{[31]}$. Another report demonstrated that EGFR activation may be the potential determinant of primary resistance of HCC cells to sorafenib, suggesting that the overexpression of EGFR or ligand in HCC cells may lead to

Table 1. Clinical trial of sorafenib combined with TACE for intermediate stage HCC. "The level of significance for the primary endpoint TTP was set at $15 \%$, which was a significant result for sorafenib. However, the period of time until TACE ineligibility was $95 \mathrm{~d}$ for sorafenib and $224 \mathrm{~d}$ for placebo [HR 1.586 (95\% Cl 1.200-2.096), $P=0.999$ ], and as there was a good result with placebo, the efficacy of the TACE and sorafenib combination therapy was not promising.

\begin{tabular}{|c|c|c|c|c|}
\hline Trials & Design & Phase & Primary endpoint & \\
\hline SPACE ${ }^{[27]}$ & TACE+sorafenib vs TACE+placebo (TACE; DEB/scheduled) & $r-I I$ & $\begin{array}{l}\text { Median TTP } 169 \text { vs } 166 \mathrm{~d} \\
\text { HR } 0.79,95 \% \mathrm{Cl} 0.588-1.080, P=0.072\end{array}$ & * \\
\hline Post-TACE ${ }^{[28]}$ & $\begin{array}{l}\text { TACE+sorafenib vs TACE+placebo (TACE; conventional/1 or } \\
2 \text { sessions) }\end{array}$ & III & $\begin{array}{l}\text { Median TTP } 5.4 \text { vs } 3.7 \text { months } \\
\text { HR } 0.87,95 \% \mathrm{Cl} 0.70-1.09, P=0.252\end{array}$ & Negative \\
\hline NCT010004978 (ECOG1208) & $\begin{array}{l}\text { TACE+sorafenib vs TACE+placebo (TACE; conventional or } \\
\text { DEB/scheduled) }\end{array}$ & III & PFS & Ongoing \\
\hline NCT01324076 (TACE-2) & TACE+sorafenib vs TACE+placebo (TACE; DEB/on demand) & III & PFS & Ongoing \\
\hline NCT01217034 (TACTICS) & $\begin{array}{l}\text { TACE+sorafenib vs TACE alone (TACE; conventional/on } \\
\text { demand) }\end{array}$ & II & Time to untreatable progression & Ongoing \\
\hline
\end{tabular}


sustained activation of EGFR downstream signaling and drug resistance to sorafenib ${ }^{[32]}$.

In addition to EGFR and its ligand, downstream signaling molecules, especially Ras/Raf/MEK/ERK, might contribute to sorafenib resistance. In HCC, the Ras/Raf/MEK/ERK signaling pathway is often activated, and the MAPK level affects the sensitivity of HCC to sorafenib. Studies have found that down-regulation of pERK may be associated with HCC sorafenib resistance ${ }^{[33]}$. It has recently been reported that the c-Jun N-terminal kinase (JNK), another member of the MAPK family, can serve as a biomarker to predict the sensitivity to sorafenib ${ }^{[34]}$.

VEGFR is also one of sorafenib's cellular targets. Studies have shown that VEGFA stimulates paracrine secretion of hepatocyte growth factor by stromal cells, which induces tumor progression ${ }^{[35]}$. HCC patients with VEGFA amplification are distinctly sensitive to sorafenib, indicating that VEGFA might be a candidate predictor of sorafenib response in HCC.

Thus, it is urgent to identify predictive biomarkers for primary resistance to sorafenib, and then apply the concept of individualized treatment or seek therapeutic strategies such as combining sorafenib with other anticancer agents to treat HCC.

\section{Acquired resistance}

\section{PI3K/Akt pathway and sorafenib resistance}

PI3K/Akt is an important pathway involving cell apoptosis and chemotherapeutic drug resistance. By inhibiting the expression of Akt, we can sensitive cells to sorafenib-induced apoptosis ${ }^{[36]}$. It has been reported that sorafenib can activate SHP-1 and then negatively regulate the expression of pSTAT3 and inhibit the JAK/STAT signaling pathway. In acquired drug-resistant HCC cell lines, we identified abnormal changes in the JAK/STAT pathway, such as high expression of pSTAT3 and its downstream pro-apoptotic proteins, Mcl-1 and cyclin D1, and lowered the expression of SHP-1 and pSHP- $1^{[37]}$, suggesting that sorafenib-related resistance may partly result from the abnormal activation of STAT3.

\section{Autophagy and sorafenib resistance}

Autophagy is the body's self-protective mechanism under various stress-induced signals, and currently, its role in HCC cells is quite controversial. This mechanism may promote cancer growth because it enables cells to survive nutrient deprivation. Shimizu et $a l^{[38]}$ found that sorafenib treatment led to accumulation of autophagosomes and activation of autophagic flux, as evidenced by increased LC3 lipidation and a clear decline of the autophagy substrate p62 in Huh7, HLF and PLC/ $\mathrm{PRF} / 5$ cells, thus promoting HCC cell survival and limiting sorafenib efficiency. However, by using chloroquine, 3-MA or RNA interference that targets autophagy-related genes, the anti-tumor effect of sorafenib was significantly improved. In addition, studies have demonstrated that autophagy induced by PSMD10, also known as gankyrin or p28, promotes tumor progression $^{[39]}$. PSMD10 augmented autophagic flux to resist sorafenib or conventional chemotherapy, and inhibition of autophagy suppressed PSMD10-mediated resistance. Nevertheless, excessive stimulation may lead to programmed cell death instead of survival ${ }^{[40]}$. Several studies have also shown that excessive autophagy can promote apoptosis in tumor cells and decrease tumor size. When sorafenib was combined with pemetrexed, a folate anti-metabolite that stimulates autophagy, the treatment increased the rate of autophagy and cell death in vitro and suppressed tumor growth in vivo ${ }^{[41]}$. Thus, autophagy can either enable cell survival or promote cell death $^{[42]}$, and further in-depth research is necessary for clarification.

\section{Epithelial-mesenchymal transition and sorafenib resistance}

Epithelial-mesenchymal transition (EMT) can occur in tumor cells under conditions of hypoxia and other stimulating factors. In cancer, EMT is associated with poor patient survival $^{[43,44]}$ because it is a key step in the development of metastasis. In EMT, cell adhesion molecules (such as E-cadherin) are lost, and mesenchymal cell markers such as vimentin (VIM) are gained, resulting in the loss of polarity and cell-to-cell contacts and enhancement of tumor cell migration and invasion. Therefore, the tumor cells become more motile and insensitive to antitumor drugs, including sorafenib ${ }^{[45-47]}$. In HCC, different studies have demonstrated that sorafenib resistance mechanisms may involve $\mathrm{EMT}^{[45,48]}$. In a study conducted by van Malenstein and colleagues ${ }^{[45]}$, five resistant human liver cell lines were developed through long-term exposure to sorafenib. The cells changed in appearance, lost E-cadherin and KRT19 and showed high expression of vimentin, indicating epithelialto-mesenchymal transition. Resistant cells showed reduced adherent growth, became more invasive and lost liver-specific gene expression. However, the sensitivity to sorafenib after development of resistance can partially be restored using PI3K/Akt- or BCRP/Hedgehog-inhibitors in vitro. In addition to hepatocytes, noncellular tumor components may also play a role ${ }^{[49]}$. They manipulate hepatocellular carcinoma invasion and metastasis by facilitating epithelial-mesenchymal transition, increasing the proteolytic activity of matrix metalloproteinases, and regulating antitumor immunity. Although the exact mechanism between EMT and sorafenib resistance is still unknown, and it is uncertain whether EMT is the trigger or the result, further study investigating strategies for restoration of sensitivity to sorafenib are needed.

\section{Tumor microenvironment and sorafenib resistance}

The tumor microenvironment plays an important role in the occurrence and development of tumors. Anti-angiogenic drugs can cause tumor blood vessel contraction and reduce blood flow, resulting in a lack of oxygen to the tumor. It is widely accepted that hypoxia in solid tumors is associated with chemotherapy failure, selection of more invasive and resistant clones, and poor prognosis ${ }^{[50,51]}$. Hypoxic cells inside solid tumors are extremely resistant to therapies, as their survival ability is increased due to the cellular adaptive response to hypoxia, which is primarily controlled by hypoxia induc- 
ible factor-1a $(\mathrm{HIF}-1 \mathrm{a})^{[52]}$. HIF-1a is the upstream inducer of VEGF, which plays a key role in tumor angiogenesis. It is also a transcription factor that mediates the adaptive responses of tumor cells to hypoxia by regulating a series of genes implicated in glucose uptake, metabolism, and cell proliferation, thereby limiting sorafenib efficiency and inducing drug resistance $^{[53]}$. Studies have demonstrated that expression of these genes may result in the expansion of cells that may exhibit a drug-resistant phenotype due to altered biochemical pathways $^{[53]}$. For example, HIF-1a protein was significantly stabilized in HCC cells under hypoxia, which led to activation of the MDR1 (multidrug resistance 1) gene ${ }^{[54]}$. MDR1 encodes for P-gp (P-glycoprotein), which can decrease the intracellular concentration of chemotherapeutic drugs, including sorafenib $^{[55]}$. HIF-1 costabilization can also be enhanced when ADRB2 signaling negatively regulates autophagy ${ }^{[56]}$, leading to reprogramming of glucose metabolism in hepatocellular carcinoma cells and the acquisition of resistance to sorafenib.

In addition to HIF-1a, HIF-2a may also play a role in sorafenib resistance. Sorafenib inhibits HIF-1a synthesis, resulting in the hypoxic response switch from HIF-1a- to HIF2a-dependent pathways and providing a mechanism for more aggressive growth of tumors. The present study has demonstrated that up-regulation of HIF-2a induced by sorafenib contributes to the resistance of hypoxic HCC cells by activating the transforming growth factor (TGF)-a/epidermal growth factor receptor (EGFR) pathway ${ }^{[57]}$. Hypoxia is also associated with up-regulation of the CXCL12/CXCR4/CXCR7 chemokine axis, and the CXCL12/CXCR4/CXCR7 chemokine axis can activate the ERK/MAPK and JAK/STAT signaling pathways, promoting the progression of malignant tumors and inducing drug resistance to sorafenib ${ }^{[58]}$.

\section{Epigenetic regulation involved in drug resistance}

Abundant evidence suggests that the occurrence and development of HCC is closely related to epigenetics. DNA methylation, histone modification, aberrant expression of miRNAs and dysregulated expression of many epigenetic regulatory genes occur in HCC.

It has been suggested that histone modifications orchestrate with DNA methylation to regulate the expression of genes. Studies have demonstrated that the expression of some histone methyltransferases, such as EZH2 ${ }^{[59]}$, contribute to the epigenetic silencing of target genes that regulate cancer cell growth and survival, which have been associated with sorafenib resistance of HCC cells. EZH2 knockdown or EZH2 inhibition treatment promoted sorafenib-induced hepatoma cell growth arrest and apoptosis.

MicroRNAs (miRNAs) are known to play a part in regulating important cellular processes. They generally perform their regulatory function through binding with target mRNAs, ultimately leading to repression of target protein expression levels. However, studies ${ }^{[60]}$ have demonstrated that aberrant levels of miRNAs, including miR-181a, are found in hepatocellular carcinoma (HCC). Azumi et al ${ }^{[61]}$ found that miR-181a directly targets RASSF1, a MAPK signaling factor, and knock- down of RASSF1 increased sorafenib resistance. MiR-429 ${ }^{[62]}$ promotes liver tumour-initiating cell properties by targeting $\mathrm{Rb}$ binding protein 4 , and the ectopic expression of miR-484 ${ }^{[63]}$ initiates tumourigenesis and cell malignant transformation through the synergistic activation of the transforming growth factor- $\beta /$ Gli and nuclear factor- $\mathrm{kB} /$ type I IFN pathways to enhance chemotherapeutic resistance and increase tumorigenicity.

\section{Other possible mechanisms}

Cancer stem cells may also play an important role in sorafenib resistance. Label-retaining cancer cells (LRCCs) are a subset of cancer stem cells. Xin et $a l^{[64]}$ found that after sorafenib treatment, LRCCs are highly enriched in hepatocellular carcinoma cells, which displayed more resistance to sorafenib-induced cell toxicity and apoptosis through continuous activation of ERK or Akt signaling.

Shp2 (Src homology 2 domain-containing phosphatase 2), a tyrosine phosphatase previously known as a pro-leukemogenic molecule, is also known to promote liver cancer progression and to suppress sorafenib treatment response. Studies $^{[65]}$ have demonstrated that Shp2 promotes HCC growth and metastasis by coordinately activating the Ras/Raf/Erk pathway and PI3K/Akt/mTOR cascade. Moreover, downregulation of Shp2 enhanced the sensitivity of hepatoma cells to sorafenib treatment, and patients with low Shp2 expression exhibited a superior response to sorafenib' treatment, implying that Shp2 might be a potential biomarker for sorafenib response.

Sorafenib resistance mechanisms may also be associated with the up-regulation of FGF signaling pathways. There are three signal transduction pathways in tumor angiogenesis: VEGFR (vascular endothelial growth factor receptor), PDGFR (platelet-derived growth factor receptor), and FGF (fibroblast growth factor) tyrosine kinase receptor-related pathways. Allen et $a l^{[66]}$ established a biological pancreatic tumor model and found that the use of a VEGFR antibody inhibited tumor growth in the initial stage. Then, a VEGF-independent angiogenic signaling pathway was significantly up-regulated, which led to the occurrence of drug resistance. However, at this time, dual inhibitors of FGF/VEGF can still control progression and reduce recurrence and metastasis of tumors. In addition, screening for liver cancer genes showed that FGF19 is an oncogene in $\mathrm{HCC}^{[67]}$. Therefore, it is necessary to conduct more in-depth studies to determine whether the sorafenib drug-resistance mechanism is associated with the up-regulation of FGF signaling pathways.

\section{Emerging theory}

An emerging theory to explain the resistance to anti-angiogenic drugs, including sorafenib, is 'vessel co-option,' ie, the ability of tumors to hijack the existing vasculature in organs such as the lungs or liver, thus limiting the need for angiogenesis $^{[68]}$. Vessel co-option has been reported in liver metastases ${ }^{[69]}$. Kuczynski and colleagues ${ }^{[70]}$ used an orthotopic human HCC model and found that up to $75 \%(610.9 \%)$ of the 
total vessels were provided by vessel co-option in resistant tumors relative to $23.3 \%(610.3 \%)$ in untreated controls. This is the first documentation of vessel co-option as a mechanism of acquired resistance to anti-angiogenic therapy and could have important implications, including potential therapeutic benefits derived from targeting vessel co-option in conjunction with vascular endothelial growth factor receptor signaling.

\section{Conclusion and future development}

The overall treatment outcomes for liver cancer are far from satisfactory. The use of sorafenib has been hampered by side effects. Adverse events that were reported for patients receiving sorafenib were predominantly gastrointestinal, constitutional, or dermatologic in nature, including diarrhea, weight loss, hand-foot skin reaction, alopecia, anorexia, and voice changes. In severe cases, it can lead to hypertension, abdominal pain and even discontinuation of therapy. In addition, due to the genetic heterogeneity of HCC, some patients are initially resistant to sorafenib, which has led to research to identify predictive biomarkers for primary resistance to sorafenib. It has recently been reported that basal pERK levels, JNK and VEGFA may be candidate predictors for sorafenib response in HCC. Moreover, several mechanisms are involved in the acquired resistance to sorafenib, such as crosstalk involving PI3K/Akt and JAK-STAT pathways, the activation of hypoxiainducible pathways, and epithelial-mesenchymal transition. (Figure 4).

\section{Acknowledgements}

This work was supported by the State Key Project for Liver Cancer (2012ZX10002-009), the National Research Program of China (2012CB316503, 2012AA02A201), the National Natural Science Foundation of China (81301811, 81422032, 81300306, 81372674,81672860 and 91529303 ) and the Science Foundation of Shanghai (134119a3700).

\section{References}

1 Llovet JM, Ricci S, Mazzaferro V, Hilgard P, Gane E, Blanc JF, et al. SHARP Investigators Study Group. Sorafenib in advanced hepatocellular carcinoma. N Engl J Med 2008; 359: 378-90.

2 Blumenschein GR Jr, Gatzemeier U, Fossella F, Stewart DJ, Cupit L, Cihon F, et al. Phase II trial of single-agent sorafenib in patients with advanced non-small-cell lung carcinoma. J Clin Oncol 2006; 24: 364.

3 Kim A, Widemann BC, Krailo M, Jayaprakash N, Fox E, Weigel B, et al. Phase 2 trial of sorafenib in children and young adults with refractory solid tumors: a report from the Children's Oncology Group. Pediatr Blood Cancer 2015; 62: 1562-6.

4 Steinbild S, Mross K, Frost A, Morant R, Gillessen S, Dittrich C, et al. A clinical phase II study with sorafenib in patients with progressive hormone-refractory prostate cancer: a study of the CESAR Central European Society for Anticancer Drug Research-EWIV. Br J Cancer 2007; 97: 1480-5.

5 Moreno-Aspitia A, Morton RF, Hillman DW, Lingle WL, Rowland KM $\mathrm{Jr}$, Wiesenfeld $\mathrm{M}$, et al. Phase II trial of sorafenib in patients with metastatic breast cancer previously exposed to anthracyclines or taxanes: North Central Cancer Treatment Group and Mayo Clinic Trial N0336. J Clin Oncol 2009; 27: 11-5.

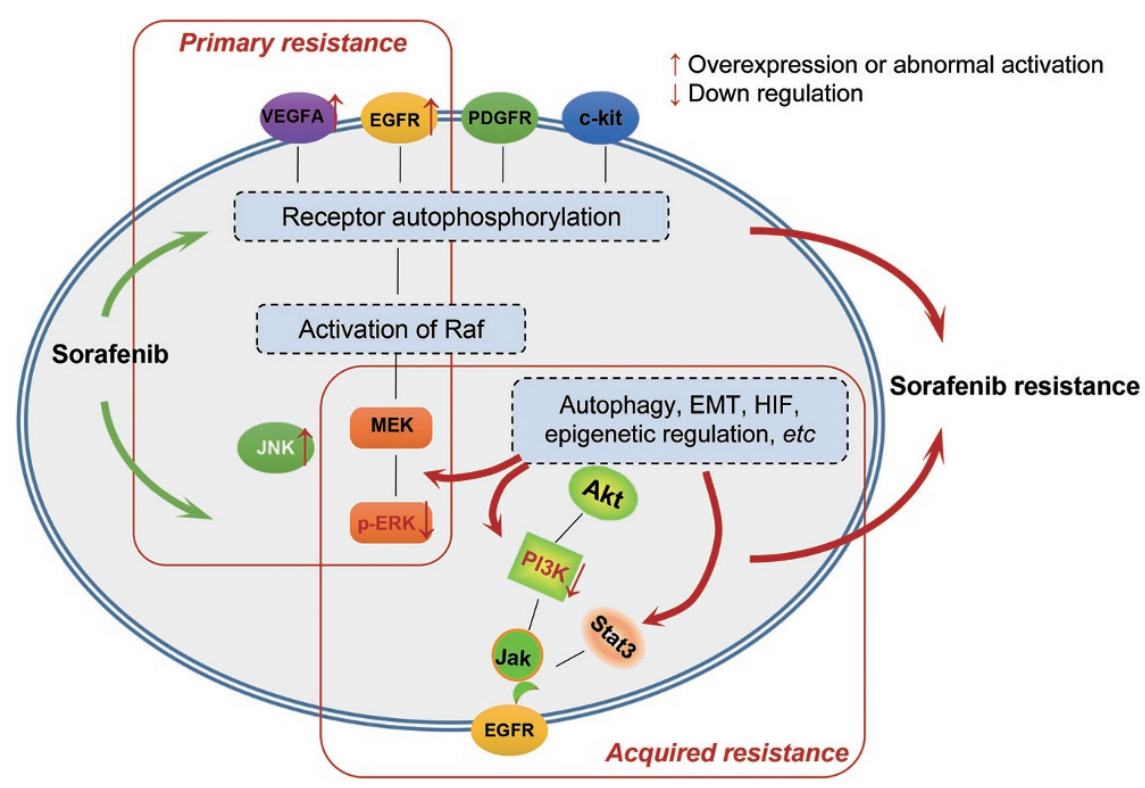

Figure 4. Mechanism of sorafenib resistance in liver cancer. Sorafenib's broad-spectrum anti-tumor effect has brought new inspiration; however, side effects and drug resistance have hampered its use clinically. How to improve the clinical efficacy has become an urgent problem. On the one hand, tumor genome sequencing technology is expected to make it possible to predict the effect of sorafenib by defining novel molecular markers. On the other hand, sorafenib combined with other molecular targeted drugs or traditional treatments may play more important role for individual treatment in future. 
6 Wilhelm SM, Carter C, Tang L, Wilkie D, McNabola A, Rong H, et al. BAY 43-9006 exhibits broad spectrum oral anti-tumor activity and targets the Raf/MEK/ERK pathway and receptor tyrosine kinases involved in tumor progression and angiogenesis. Cancer Res 2004; 64: 7099-109.

7 Tanaka S, Arii S. Molecular targeted therapiies in hepatocellular carcinoma. J Semin Oncol 2012; 39: 486-92.

8 Carlomagno F, Anaganti S, Guida T, Salvatore G, Troncone G, Wilhelm $\mathrm{SM}$, et al. BAY 43-9006 inhibition of oncogenic RET mutants. J Natl Cancer Inst 2006; 98: 326-34.

9 Sharma A, Trivedi NR, Zimmerman MA, Tuveson DA, Smith CD, Robertson GP. Mutant V599EB-Raf regulates growth and vascular development of malignant melanoma tumors. Cancer Res 2005; 65: 2412-21.

10 Wilhelm S, Carter C, Lynch M, Lowinger T, Dumas J, Smith RA, et al. Discovery and development: a multikinase inhibitor for treating cancer. Nat Rev Drug Discov 2006; 5: 835-44.

11 Centers for Disease Control and Prevention (CDC). Hepatocellular carcinoma-United States 2001-2006. Morb Mortal Wkly Rep 2010; 59: 517-20.

12 Maluccio M, Covey A. Recent progress in understanding, diagnosing, and treating hepatocellular carcinoma. CA Cancer J Clin 2012; 62: 394-9.

13 Cheng AL, Kang YK, Chen Z, Tsao CJ, Qin S, Kim JS, et al. Efficacy and safety of sorafenib in patients in the Asia-Pacific region with advanced hepatocellular carcinoma: a phase III randomised, double-blind, placebo-controlled trial. Lancet Oncol 2009; 10: 25-34.

14 Hampton T. Cancer drug trials show modest benefit: drugs target live, gastric, head and neck cancers. JAMA 2007; 298: 273-5.

15 DeVita VT Jr, Abou-Alfa GK. Therapeutic implications of the new biology. Cancer J 2000; 6: S113-20.

16 Sui ZG, Xue HW, Jing FB, Leng P. Sorafenib plus Capecitabine for patients with advanced hepatocellular caicinoma. China Pharmacy (ZhongGuo Yao Fang) 2008; 19: 848-9.

17 Awada A, Gil T, Whenham N, Van Hamme J, Besse-Hammer T, Brendel $E$, et al. Safety and pharmacokinetics of sorafenib combined with capecitabine in patients with advanced solid tumors: results of a phase I trial. J Clin Pharmacol 20011; 5: 271.

18 Abou-Alfa GK, Johnson P, Knox JJ, Capanu M, Davidenko I, Lacava J, et al. Doxorubicin plus sorafenib vs doxorubiein alone in patients with advanced hepatocellular carcinoma: a randomized trial. JAMA 2010; 304: 2154-60.

19 Richly H, Kupsch P, Passage K, Grubert M, Hilger RA, Voigtmann $\mathrm{R}$, et al. Results of a phase I trial of BAY43-9006 in combination with doxorubicin in patients with primary hepatic cancer. Int J Clin Pharmacol Ther 2004; 42: 650-1.

20 Takimoto $\mathrm{CH}$, Awada A. Safety and anti-tumor activity of sorafenib in combination with other anti-cancer agents: a review of clinical trials. Cancer Chemother Pharmacol 2008; 61: 535-48.

21 Hsu CH, Shen YC, Lin ZZ, Chen PJ, Shao YY, Ding YH, et al. Phase II study of combining sorafenib with metronomic tegafur/uracil for advanced hepatocellular carcinoma. J Hepatol 2010; 53: 126-31.

22 Prete SD, Montella L, Caraglia M, Maiorino L, Cennamo G, Montesarchio $\mathrm{V}$, et al. Sorafenib plus octreotide is an effective and safe treatment in advanced hepatocellular carcinoma: multicenter phase II So.LAR.study. Cancer Chemother Pharmacol 2010; 66: 837-44.

23 Liu X, Liu WF. Imaging evaluation on the curative effect of radiofrequency ablation for primary hepatic carcinoma. Chin J Interv Imaging Ther 2012; 9: 136-8.

24 Sun JJ, Zhao HJ, Li W. Clinical study of radiofrequency ablation therapy in combination with sorafenib for advanced hepatocellular carcinoma. J Clin Hepatol 2011; 27: 1093-8.

25 Chao Y, Chung YH, Han G, Yoon JH, Yang J, Wang J, et al. Study in Asia of the combination of transarterial chemoembolization (TACE) with sorafenib in hepatocellular carcinoma trial (START): second interim safety and efficacy analysis. J Clin Oncol 2010; 28: 4026.

26 Bruix J, Takayama T, Mazzaferro V, Chau GY, Yang J, Kudo M, et al. A phase 3, randomised, doubleblind, placebo-controlled trial of adjuvant sorafenib for hepatocellular carcinoma after resection or ablation (STORM). Lancet Oncol 2015; 16: 1344-54.

27 Lencioni R, Llovet JM, Han G, Tak WY, Yang J, Guglielmi A, et al. Sorafenib or placebo plus TACE with doxorubicin-eluting beads for intermediate stage HCC: the SPACE trial. J Hepatol 2016; 64: 10908.

28 Kudo M, Imanaka K, Chida N, Nakachi K, Tak WY, Takayama T, et al. Phase III study of sorafenib after transarterial chemoembolisation in Japanese and Korean patients with unresectable hepatocellular carcinoma. Eur J Cancer 2011; 47: 2117-27.

29 O'Connor R, Clynes M, Dowling P, O'Donovan N, O'Driscoll L. Durg sesistance in cancer-searching for mechanism, markers and therapeutic agents. Expert Opin Drug Metab Toxicol 2007; 3: 805-17.

30 Ito Y, Takeda T, Sakon M, Tsujimoto M, Higashiyama S, Noda K, et al. Expression and clinical significance of erb-B receptor family in hepatocellular carcinoma. J Cancer 2001; 84: 1377-83.

31 Blivet-Van Eggelpoël MJ, Chettouh H, Fartoux L, Aoudjehane L, Barbu V, Rey $\mathrm{C}$, et al. Epidermal growth factor receptor and HER-3 restrict cell response to sorafenib in hepatocellular carcinoma cells. J Hepatol 2012; 57: 108-15.

32 Ezzoukhry Z, Louandre C, Trécherel E, Godin C, Chauffert B, Dupont S, et al. EGFR activation is a potential determinant of primary resistance of hepatocellular carcinoma cells to sorafenib. Int J Cancer 2012; 131: 2961-9.

33 Zhang Z, Zhou X, Shen H, Wang D, Wang Y. Phosphorylated ERK is potential predictor of sensitivity to sorafenib when treating hepatocellular carcinom: evidence from an in vitro study. BMC Med 2009; 7: 41.

34 Hagiwara S, Kudo M, Nagai T, Inoue T, Ueshima K, Nishida N, et al. Activation of JNK and high expression level of CD133 predict a poor response to sorafenib in hepatocellularcarcinoma. $\mathrm{Br} J$ Cancer 2012; 106; 1997-2003.

35 Llovet JM. Focal gains of VEGFA: candidate predictors of sorafenib response in hepatocellular carcinoma. Cancer Cell 2014; 25: 560-2.

36 Morgensztern D, McLeod HL. PI3K/Akt/mTOR pathway as a target for cancer therapy. Anticancer Drugs 2005; 16: 797-803.

37 Chen KF, Tai WT, Hsu CY, Huang JW, Liu CY, Chen PJ, et al. Blockade of STAT3 activation by sorafenib derivatives through enhancing SHP-1 phosphatase activity. Eur J Med Chem 2012; 55: 220-7.

38 Shimizu S, Takehara T, Hikita H, Kodama T, Tsunematsu H, Miyagi $\mathrm{T}$, et al. Inhibition of autophagy potentiates the antitumor effect of the multikinase inhibitor sorafenib in hepatocellular carcinoma. Int J Cancer 2012; 131: 548-57.

39 Luo T, Fu J, Xu A, Su B, Ren YB, Li N, et al. PSMD10/gankyrin induces autophaty to promote tumor progression through cytoplasmic interaction with ATG7 and nuclear transactivation of ATG7 expression. Autophagy 2016; 12: 1355-71.

40 Tsujimoto Y, Shimizu, S. Another way to die: autophagic programmed cell death. Cell Death Differ 2005; 12: 1528-34.

41 Bareford MD, Hamed HA, Tang Y, Cruickshanks N, Burow ME, Fisher $\mathrm{PB}$, et al. Sorafenib enhances permetrexed cytotoxicity through an autophagy -dependent mechanism in cancer cells. Autophagy 2011; 7: 1261-2. 
42 Heqing Y, Bin L, Xuemei Y, Linfa L. The role and mechanism of autophagy in sorafenib targeted cancer therapy. Crit Rev Oncol Hematol 2016; 100: 137-40.

43 Kalluri R, Weinberg RA. The basics of epithelial-mesenchymal transition. J Clin Invest 2009; 119: 1420-8.

44 Marcucci F, Stassi G, De Maria R. Epithelial-mesenchymal transition: a new target in anticancer durg didcovery. Nat Rev Drug Discov 2016; 15: 311-25.

45 van Malenstein H, Dekervel J, Verslype C, Van Cutsem E, Windmolders $\mathrm{P}$, Nevens $\mathrm{F}$, et al. Long-term exposure to sorafenib of liver cancer cells induces resistance with epithelial-to-mesenchymal transition, increased invasion and risk of rebound growth. Cancer Lett 2013; 329: 74-83.

46 Kalluri R, Weinberg RA. The basics of epithelial-mesenchymal transition. J Clin Invest 2009; 119: 1420-8.

47 Maheswaran T, Rushbrook SM. Epithelial-mesenchymal transition and the liver: role in hepatocellular carcinoma and liver fibrosis. J Gastroenterol Hepatol 2012; 27: 418-20.

48 Dazert E, Colombi M, Boldanova T, Moes S, Adametz D, Quagliata L, et al. Quantitive proteomics and phosphoproteomics on serial tumor biopsies from a sorafenib-treated HCC patient. Proc Natl Acad Sci U S A 2016; 113: 1381-6.

49 Wang HY, Chen L. Tumor microenviroment and hepatocellular carcinoma metastasis. J Gastroenterol Hepatol 2013; 28: 43-8.

50 Trédan O, Galmarini CM, Patel K, Tannock IF. Drug resistance and the solid tumor microenvironment. J Natl Cancer Inst 2007; 99: 144154.

51 Tak E, Lee S, Lee J, Rashid MA, Kim YW, Park JH, et al. Human carbonyl reductase 1 upregulated by hypoxia renders resistance to apoptosis in hepatocellular carcinoma cells. J Hepatol 2011; 54: 328-39.

52 Powis G, Kirkpatrick L. Hypoxia inducible factor-1alpha as a cancer drug target. Mol Cancer Ther 2004; 3: 647-54.

53 Liu LP, Ho RL, Chen GG, Lai PB. Sorafenib inhibits hypoxia-inducible factor- $1 \alpha$ synthesis: implications for antiangiogenic activity in hepatocellular carcinoma. Clin Cancer Res 2012; 18: 5662-71.

54 Comerford KM, Wallace TJ, Karhausen J, Louis NA, Montalto MC, Colgan SP. Hypoxia-inducible factor-1-dependent regulation of the multidrug resistance (MDR1) gene. Cancer Res 2002; 62: 3387-94.

55 Poller B, Wagenaar E, Tang SC, Schinkel AH. Double-transduced MDCKII cells to study human P-glycoprotein (ABCB1) and breast cancer resistance protein (ABCG2) interplay in drug transport across the blood-brain barrier. Mol Pharm 2011; 8: 571-82.

56 WuFQ, FangT, Yu LX, Lv GS, Lv HW, Liang D, et al. ADRB2 signaling promotes HCC progression and sorafenib re sistance by inhibiting autoph agic degradatio $n$ of HIF1a. J Hepatol 2016; 65: 314-24.

57 Zhao D, Zhai B, He C, Tan G, Jiang X, Pan S, et al. Upregulation of HIF$2 \alpha$ induced by sorafenib contributes to the resistance by activating the TGF- $\alpha$ /EGFR pathway in hepatocellular carcinoma cells. Cell Signal 2014; 26: 1030-9.

58 Monnier J, Boissan M, L'Helgoualc'h A, Lacombe ML, Turlin B, Zucman-Rossi J, et al. CXCR7 is up-regulated in human and murine hepatocellular carcinoma and is specifically expressed by endothelial cells. Eur J Cancer 2012; 48: 138-48.

59 Wang S, Zhu Y, He H, Liu J, Xu L, Zhang H, et al. Sorafenib suppress growth and survival of hepatoma cells by accelerating degradation of zeste homolog 2. Cancer Sci 2013; 104: 750-9.

60 Tan JY, Habib NA, Chuah YW, Yau YH, Geifman-Shochat S, Chen WN. Identification of cellular targets of microRNA-181a in HepG2 cells: a new approach for functional analysis of microRNAs. PLoS One 2015; 10: $123-67$.

61 Azumi J, Tsubota T, Sakabe T, Shiota G. miR-181a induces sorafenib resistance of hepatocellular carinoma cells through downregulation of RASSF1 expression. Cancer Sci 2016; 6: 62-71.

62 Li L, Tang J, Zhang BH, Yang W, Liu MY,Wang RY, et al. Epigenetic modification of MiR-429 promotes liver tumour-initiating cell properties by targeting Rb binding protein 4. Hepatology 2014; 2; 11-23.

63 Yang YC, Lin XM, Lu XY, Luo GJ, Zeng T, Tang J, et al. Interferon-microRNA signalling drives liver precancerous lesion formation and hepatocarcinogenesis. Gut 2016; 65: 1186-201.

64 Xin HW, Ambe CM, Hari DM, Wiegand GW, Miller TC, Chen JQ, et al. Label-retaining liver cancer cells are relatively resistant to sorafenib. Gut 2013; 62: 1777-86.

65 Han T, Xiang DM, Sun W, Liu N, Sun HL, Wen W, et al. PTPN11/Shp2 overexpression enhances liver cancer progression and predicts poor prognosis of patients. J Hepatol 2015; 63: 651-60.

66 Allen E, Walters IB, Hanahan D. Brivanib, a dual FGF/VEGF inhibitor, is active both first and second line against mouse pancreatic neuroendocrine tumors developing adaptive/evasive resistance to VEGF inhibition. Clin Cancer Res 2011; 17: 5299-310.

67 Sawey ET, Chanrion M, Cai C, Wu G, Zhang J, Zender L, et al. Identification of a therapeutic strategy targeting amplified FGF19 in liver cancer by Oncogenomic screening. Cancer Cell 2011; 19: 347-58.

68 Donnem T, Hu J, Ferguson M, Adighibe O, Snell C, Harris AL, et al. Vessel co-option in primary human tumors and metastases: an obstacle to effective anti-angiogenic treatment? Cancer Med 2013; 2: 427-36.

69 Vermeulen PB, Colpaert C, Salgado R, Royers R, Hellemans H, Van Den Heuvel E, et al. Liver metastases from colorectal adenocarcinomas grow in three patterns with different angiogenesis and desmoplasia. J Pathol 2001; 195: 336-42.

70 Kuczynski EA, Yin M, Bar-Zion A, Lee CR, Butz H, Man S, et al. Cooption of liver vessels and not sprouting angiogenesis drives acquired sorafenib resistance in hepatocellular carcinoma. J Natl Cancer Inst 2016; 108. pii: djw030. 